\title{
Corrigendum: Observation and coherent control of interface-induced electronic resonances in a field-effect transistor
}

\author{
J. O. Tenorio-Pearl, E. D. Herbschleb, S. Fleming, C. Creatore, S. Oda, W. I. Milne and A. W. Chin
}

Nature Materials 16, 208-213 (2017); published online 19 September 2016; corrected after print 30 June 2017

In this work we reported the development of a rigorous mathematical and physical framework in order to model the detailed time-resolved behaviour of over 800 resonances that we studied, through continuous-wave and single-pulse microwave spectroscopy measurements in a field-effect transistor. It has been pointed out to us that we omitted citations to the following works, which report continuous-wave measurements of high-Q resonances in similar devices: T. Ferrus et al., J. Appl. Phys. 106, 033705 (2009); A. Rossi \& D. G. Hasko, J. Appl. Phys. 108, 034509 (2010); M. Erfani, D. G. Hasko, A. Rossi, W. S. Cho \& J.-B. Choi, Appl. Phys. Lett. 99, 192108 (2011). The observations were assigned by the authors to spatial Rabi oscillations of trapped electrons. In our work, the experimental evidence that is currently available cannot unambiguously assign the microscopic origin of the observed resonances (see conclusions). 